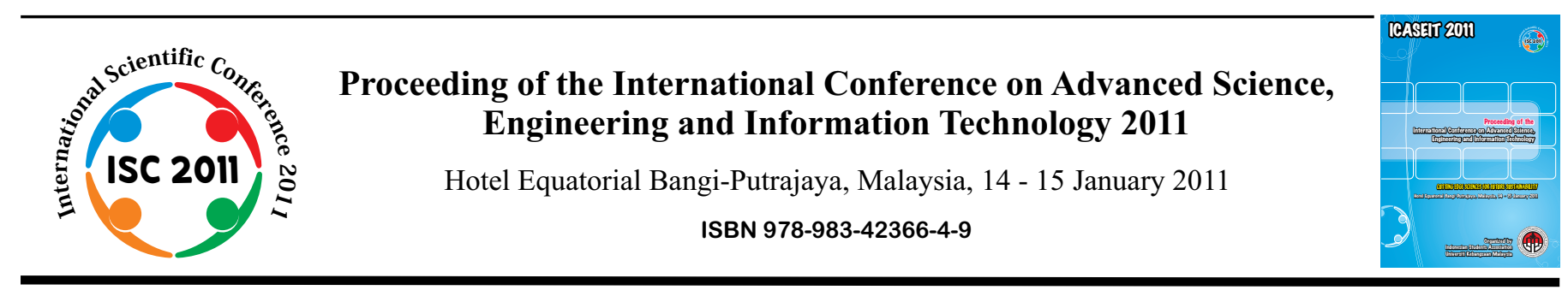

\title{
Effect of Rain Attenuations on Free Space Optic Transmission in Kuala Lumpur
}

\author{
Fatin Hamimi Mustafa ${ }^{\#}$,Abu Sahmah M Supaat ${ }^{*}$ Nachimani Charde ${ }^{\# \#}$ \\ \#, ${ }^{*}$ Photonic Technology Center \\ Faculty of Electrical Engineering, Universiti Teknologi Malaysia, Skudai, 81310 Johor \\ Tel.:+6012-7895870\#, E-mail: fatin_ferrarieyahoo.com, abus@fke.utm.my* \\ \#\# Faculty of Electrical Engineering, Nottingham University Malaysia Campus, Semenyih, 43500 Selangor \\ E-mail: nachicharde@yahoo.com
}

\begin{abstract}
This research paper describes the effects of rain attenuation on the free space optical (FSO) transmission system. The entire research was conducted in the heart of Kuala Lumpur city- year 2001, as the city was using such systems (LAN-to-LAN \& WLAN-to-WLAN) widely to connect the metropolitan areas for data transmission. The preliminary experiments were done using PAVLight product modeled PL-1G/1Tx/GigE which leaded to maximum reach of $700 \mathrm{~m}$ distance with 810nm wavelength and also laser power of $14 \mathrm{dBm}$. The data for rain intensities were obtained from measuring unit at UTM Jalan Semarak, Kuala Lumpur following the ITU protocols such as ITU-R P.1814 and ITU-R P.1817. The transmission and reception were achieved by using Continuous Wave (CW) Laser signals as transmitting signals and APD photo detector to receive it on the other end. The experiments were fully carried out for two conditions (heavy-rain and lighter rain or drizzle) with multiple repetitions. At last, the collected data were compiled and compared with other researcher's results. It was found to be the same as how the other researchers have obtained before for various cities worldwide. The results were found to be very satisfactory. It obeyed the theoretical aspects as higher the rain, higher the attenuations then. These results the degradation on the received signals. As a guide for future implementation of FSO systems for various atmospheric conditions in the metropolitan areas of Kuala Lumpur; we believed that this preliminary works may help.
\end{abstract}

Keywords - free space optical transmission system; rain attenuation; rain intensity; atmospheric conditions.

\section{INTRODUCTION}

Free-space optical system (FSO) refers to the transmission of modulated visible or infrared, IR beams through the atmosphere to achieve broadband communications. The theory of FSO is exactly the same as that of the fiber optical transmission system. The difference is that the energy beam is collimated and sent through clear air or space from the source to the destination, rather than guided through an optical fiber. An FSO system for the outdoor environment is workable over distances of several kilometers as far as it has clear line of sight (LOS) of atmosphere between the source and the destination [1]. Fig. 1 shows the FSO transmission link for outdoor environment using Line-of-Sight mechanism which creates links between transmitter and receiver. Although FSO systems seemed to be good solution for some broadband networking systems, it has the limitations. More significant factors are, it adaptable to the rain, dust, snow, fog, or smog and extras. Often can block the transmission path and shut down the network due to atmospheric changes as mentioned above.

In this paper, we examine the alteration of Continuous Wave Laser propagation over the infrared (IR) in heart of Kuala Lumpur city. Atmosphere is the conducting medium in this case, and it tends to change due to weather conditions such as rain, haze, sunny and extras [2]. The atmosphere interacts with lights due to the composition or decomposition of the atmospheric compounds and in normal conditions, it made up of variety of different molecules which is often called as aerosols [3] [4]. These molecules (moisture) are independent on size with irregularities. When the precipitation becomes more important as compared to wavelength, then the waves are attenuated by reflection and refraction [3]. In such circumstance, it can alter the rain attenuation factors as compared to before [5]. 
We have chosen the heart of Kuala Lumpur city as the testing location as to perform the analysis on real urban environment.

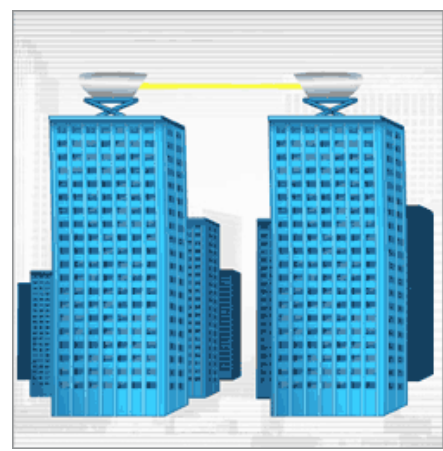

Fig.1 FSO transmission link for outdoor environment in LOS conditions between transmitter and receiver

\section{RECOMMENDATION ITU-R P.1814 AND P.1817}

Link margin or link budget is important issues in Free Space Optical system because it involves total loss of powers, expected received powers and transmitted powers [2]. The link margin $(\mathrm{dBm})$ is given as by [6];

$$
M_{\text {link }}=P_{e}-S_{r}-A_{\text {geo }}-A_{\text {atmo }}-A_{\text {syst }}
$$

Where, $M_{\text {link }}$ is link margin $(\mathrm{dB}), S_{r}$ is sensitivity, $A_{\text {geo }}$ is link geometrical attenuation, $A_{\text {atmo }}$ is atmospheric attenuation due to weather conditions such as rain, haze, fog and snow and $A_{s y s}$ represents all other system dependent losses including misalignment of the beam direction and receiver optical losses due to beam wander.

The geometric attenuations (or geometric loss) refer to losses that occurred due to the divergence of the optical beam between transmitter and receiver [7]. For Free Space Optics propagation, the beam diverges by some amount over the path from transmitter to receiver. Geometrical attenuation equation is as [6].

$$
A_{\text {geo }}(d B)=10 \log _{10}\left(\frac{S_{d}}{S_{\text {capture }}}\right)
$$

Where, $S_{\text {capture }}$ is the receiver capture surface $\left(\mathrm{m}^{2}\right), S_{d}$ is the surface area of transmit beam at range $d$, which is approximated by [6];

$$
S_{d}=\frac{\pi}{4}(d \times \theta)^{2}
$$

Where, $d$ is distance in $\mathrm{km}$ while $\theta$ is beam divergence in mrad.

The rain attenuation can be defined using the following equation [6];

$$
\gamma_{\text {rain }}=k \cdot R^{\alpha}
$$

Where $\gamma_{\text {rain }}$ is rain attenuation $(\mathrm{dB} / \mathrm{km}), \mathrm{R}$ is rain intensity $(\mathrm{mm} / \mathrm{hr}), \mathrm{k}$ and $\alpha$ is rain coefficient.

The parameters ' $\mathrm{k}$ ' and ' $\alpha$ ' depend on the rain characteristics or can be determined from measurements. Table I shows $\mathrm{k}$ and $\alpha$ parameters values used for the estimation of the specific attenuation due to rain [6] while Table II shows the International Visibility Codes for Weather Conditions and Precipitation for rain conditions [4].

TABLE I

PARAMETERS USED FOR THE ESTIMATION OF THE SPECIFIC ATTENUATION DUE TO RAIN

\begin{tabular}{|l|l|l|}
\hline Location & $\mathrm{K}$ & $\alpha$ \\
\hline Japan & 1.58 & 0.63 \\
\hline France & 1.076 & 0.67 \\
\hline
\end{tabular}

TABLE II

INTERNATIONAL VISIBILITY CODES FOR WEATHER CONDITIONS AND PRECIPITATION FOR RAIN CONDITIONS

\begin{tabular}{|l|l|l|l|}
\hline $\begin{array}{l}\text { Weather } \\
\text { Condition }\end{array}$ & Precipitation & $\begin{array}{l}\text { Amount } \\
(\mathrm{mm} / \mathrm{hr})\end{array}$ & Visibility \\
\hline Light Fog & $\begin{array}{l}\text { Cloudburst \& } \\
\text { Heavy Rain }\end{array}$ & 100 & $770 \mathrm{~m}-1 \mathrm{~km}$ \\
\hline Thin Fog & Heavy Rain & 25 & $2 \mathrm{~km}$ \\
\hline Haze & Medium Rain & 12.5 & $2.8 \mathrm{~km}-4 \mathrm{~km}$ \\
\hline Light Haze & Light Rain & 2.5 & $5.9 \mathrm{~km}-10 \mathrm{~km}$ \\
\hline Clear & Drizzle & 0.25 & $18.1 \mathrm{~km}-20 \mathrm{~km}$ \\
\hline
\end{tabular}

\section{DATA COLLECTIONS}

Malaysia has an equatorial climate, giving it a warm and wet weather due to its proximity to the equator that influence the rainfall at different intervals of the year [8] [9]. The rain intensity or rainfall rate data are the way to obtain the rain attenuations (from (4)) to be used in link margin equation (1). The measurement for rain intensity was done at UTM Jalan Semarak, Kuala Lumpur in 2001 as it shares the same telecommunication ID as compared with KLCC. Table III shows the rain intensity data for Kuala Lumpur and calculated the rain attenuations and also its precipitations. The rain attenuations are calculated (from (4)) with values of $\mathrm{k}$ and $\alpha$ are based on Japan location because of considering in Asian region. The Fig. 2 shows the graph of rain attenuations $(\mathrm{dB})$ versus rain intensity $(\mathrm{mm} / \mathrm{hr})$ for $700 \mathrm{~m}$ distance link from collected data of rain intensities and rain attenuations calculations.

\begin{tabular}{|c|c|c|c|}
\hline & $\begin{array}{l}\text { Rain } \\
\text { Intensity } \\
(\mathrm{mm} / \mathrm{hr})\end{array}$ & $\begin{array}{l}\text { Rain } \\
\text { Attenuations } \\
\text { for } 700 \mathrm{~m} \\
\text { distance }(\mathrm{dB})\end{array}$ & Precipitation \\
\hline Maximum & 174.49 & -28.581 & $\begin{array}{l}\text { Heavy Rain \& } \\
\text { Cloudburst }\end{array}$ \\
\hline Minimum & 1.040 & -1.133 & Drizzle \\
\hline Average & 36.044 & -10.108 & Heavy Rain \\
\hline
\end{tabular}

TABLE III

RAIN INTENSITY DATA FOR KUALA LUMPUR REGION, CALCULATED RAIN ATTENUATIONS AND ITS PRECIPITATIONS 


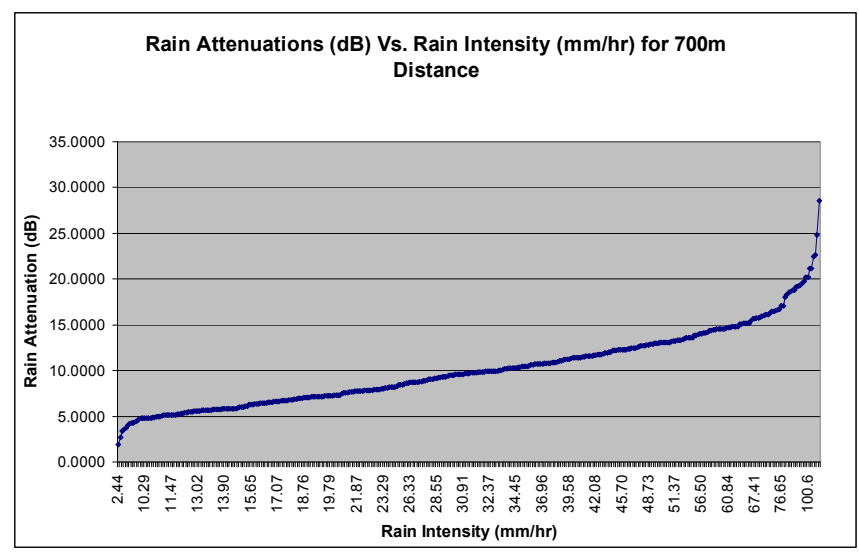

Fig. 2 The rain attenuations $(\mathrm{dB})$ versus rain intensity $(\mathrm{mm} / \mathrm{hr})$ for $700 \mathrm{~m}$ distance link

\section{Optsim Software Prediction MEASUREMENT}

\section{A. Technical Specifications}

All the parameters or technical specifications are defined by standard parameters of FSO product in the market. In this project, we use the FSO transmitter and receiver from PAVLight product for optical signals transmission. Gigabit is a free space optical system designed to enable high speed communication of voice, data and video over distances up to $700 \mathrm{~m}$ between link heads which have a clear line of sight between them. PAV Data has the worlds' largest installed system of FSO technology, with much of it being designed and deployed by their consultants. Today PAV's FSO products are providing 1000's of connectivity solutions to both the carrier and the enterprise market across the globe [10]. PAV's comprehensive product range scales from $1.54 \mathrm{Mbps}$ through to $1 \mathrm{Gbps}$, across distances up to 4 kilometers connecting at the speed of light.

Table IV shows the technical specification of FSO transmission link using PAVLight product [10]. However, based on the link margin equation (from (1) to (4)), there are some parameters not specified in PAVLight product, that of the values of received surface area and system loss. The selected typical value of received surface area is $180 \mathrm{~mm} 2$ [11] while for system loss, the typical value is $6 \mathrm{~dB}$ [12] which fulfills theoretical relationships (equation (1) to (3)).

TABLE IV

THE TECHNICAL SPECIFICATION OF FSO TRANSMISSION LINK USING PAVLIGHT PRODUCT

\begin{tabular}{|l|l|}
\hline Parameter & Specification \\
\hline Product Code & PL-1G/1Tx/GigE \\
\hline Bandwidth & $1 \mathrm{Gbps}$ \\
\hline Distance & $700 \mathrm{~m}$ \\
\hline Light Source & $\begin{array}{l}\text { Continuous Wave Laser } \\
\text { (CW Laser) }\end{array}$ \\
\hline Wavelength & $810 \mathrm{~nm}$ \\
\hline Beam Divergence & $0.003 \mathrm{rad}$ \\
\hline
\end{tabular}

\begin{tabular}{|l|l|}
\hline Transmit Power & $14 \mathrm{dBm}$ \\
\hline System Loss & $-6 \mathrm{~dB}$ \\
\hline Sensitivity & $-13 \mathrm{dBm}$ \\
\hline Photodetector & APD \\
\hline Modulation Type & Mach-Zehnder \\
\hline Receive Area & $0.0018 \mathrm{~m}^{2}$ \\
\hline
\end{tabular}

\section{B. System Design}

The FSO transmission link design consists of transmitter, atmosphere channel and receiver [13]. The transmitter of the FSO system is made with bit random sequence or PBRS, electrical signal generator, Continuous Wave Laser as optical source, external modulator and optical power normalize; before the signals have entered to atmosphere (channel) and then received at receiver.

Atmosphere (channel) is a transmission medium of FSO system where unpredictable weather condition such as haze and rain that cause of attenuation occurs. The present of noise also may occur in this channel that can degrade the signal performance. At receiver, it has optical receiver consists Avalanche Photodiode (APD), amplifier to amplify the weak signal and filter to remove the unwanted signal or to select the desired range of frequencies. Fig. 3 shows the FSO system transmission design using OPTsim software.

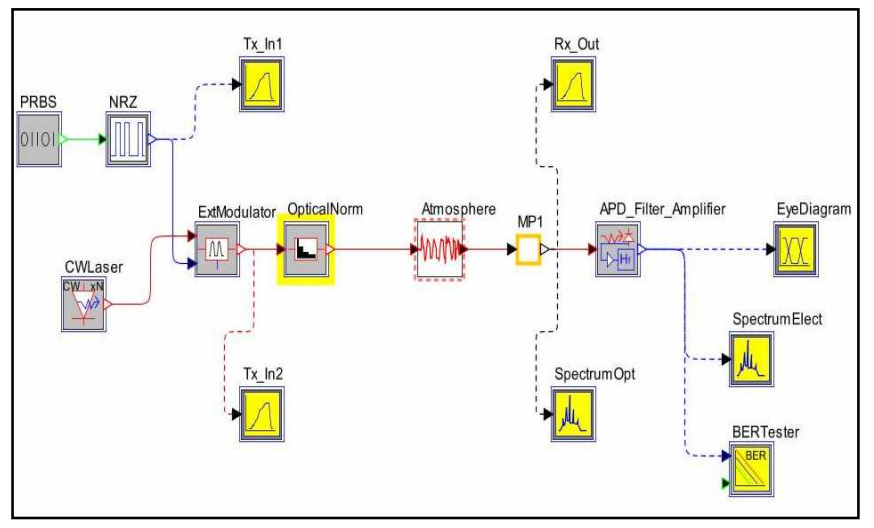

Fig. 3 The FSO system transmission design using OPTsim software

\section{RESULTS AND ANALYSIS}

The data had been plotted in OPTsim software as to see the effects of rain attenuations (conditions) in FSO transmission system. The graphs had shown the difference of the received signals between heavy rains and also drizzle. This is where the heavy rain has the higher value of rain intensity and rain attenuations compared to drizzle. The comparisons are made in order to study how was the heavy rain has affected the FSO transmission. It involves the analysis of eye diagram, bit error rate (BER) and optical 
spectrum analysis to compare the deviation between both conditions.

Fig. 4 shows the comparison of eye diagram between rain conditions for FSO transmission on maximum rain attenuations (heavy rain with cloudburst) and minimum rain attenuations (drizzle) for $700 \mathrm{~m}$ distance in FSO transmission system. Eye diagram for drizzle conditions has wider eye opening compared to heavy rain conditions because of the attenuation for heavy rain is higher than drizzle conditions, $28.6 \mathrm{~dB}$ compared to $-1.3 \mathrm{~dB}$ as mentioned earlier .

The weakness of received signal of heavy rain compared to drizzle is determined by looking the noise margin, sensitivity, timing jitter and amplitudes between both eyes diagrams [14]. The noise margin and sensitivity as compared to timing error in eye diagram for drizzle conditions is larger than for heavy rain conditions. The timing jitter of eye diagram for drizzle conditions also shows sharper than heavy rain conditions. From the eye diagram, the maximum amplitude of received signal by transmission for drizzle conditions is about $0.8 \mathrm{mV}$ and at the extent, the maximum amplitude for heavy rain conditions is about $0.15 \mathrm{mV}$. It shows the degradation on performance of signal for transmission FSO for heavy rain conditions compared to drizzle conditions due to higher rain attenuation value for heavy rain conditions in Kuala Lumpur city.

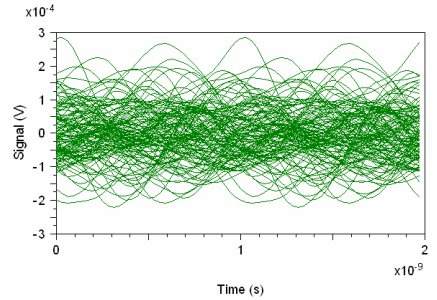

Max Rain Attenuations Rain Attenuation: -28.6dBm

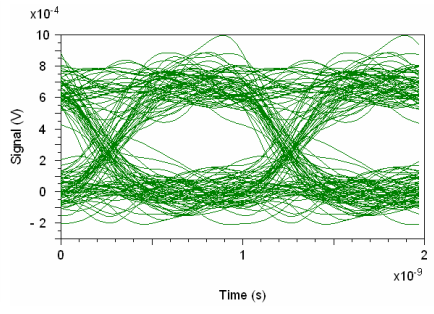

Min Rain Attenuation Rain Attenuation: $-1.3 \mathrm{~dB}$
Fig. 4 Comparison of eye diagram between rain condition for FSO transmission on maximum rain attenuations (heavy rain with cloudburst) and minimum rain attenuations (drizzle) for $700 \mathrm{~m}$ distance in FSO transmission system

The eye diagram of heavy rain conditions shows that the receive signals at receiver were distorted. It gives significant changes on the numerical values by looking at BER values. For heavy rain with cloudburst conditions, the value of BER is $10-{ }^{1}$ while for drizzle conditions; the BER value is $10-^{13}$.

The spectrum of frequencies of transmitted signals are observed on Fig. 5 and it shows the optical power $(\mathrm{dBm})$ versus wavelength $(\mathrm{m})$ of spectrum analysis for FSO transmission for maximum rain attenuations (heavy rain conditions) and meanwhile Fig. 6 shows the optical power $(\mathrm{dBm})$ versus wavelength $(\mathrm{m})$ of spectrum analysis for FSO transmission for minimum rain attenuations (drizzle conditions). By considering the link margin equation (from (1) to (4)), the link margin calculated for heavy rain with cloudburst conditions is $-66.44 \mathrm{dBm}$ or $0.227 \mathrm{nWatt}$, as proved in the Fig. 5. At the same time, the link margin calculated for drizzle conditions is $-39.14 \mathrm{dBm}$ or $121.89 \mathrm{nWatt}$, as shown in the Fig. 6.

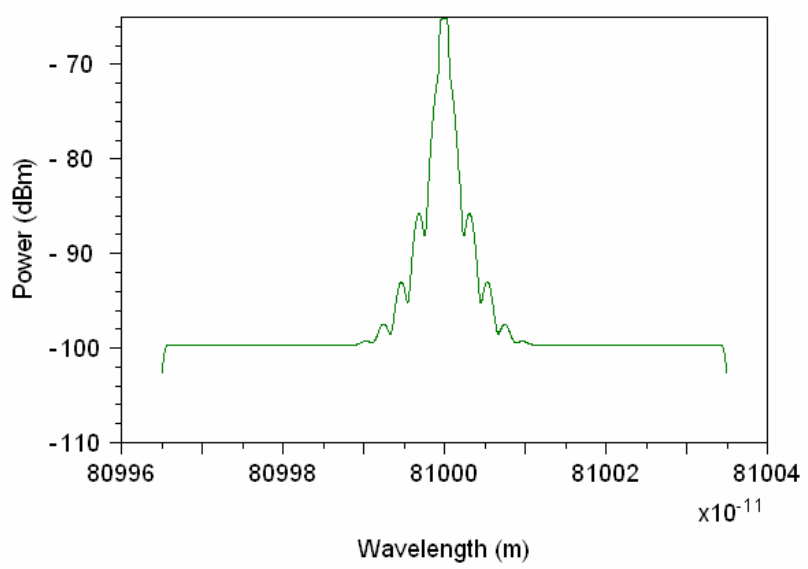

Fig. 5 Optical Spectrum for Maximum Rain Attenuations (Heavy Rain with Cloudburst Conditions)

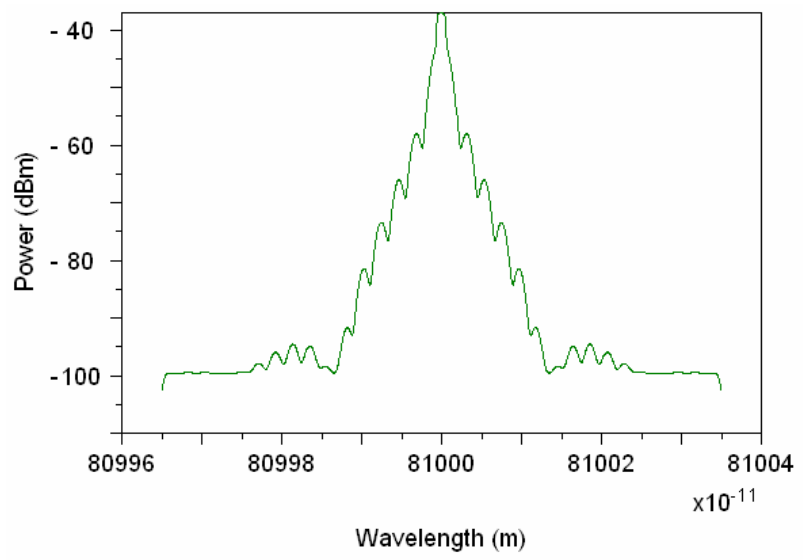

Fig.6 Optical Spectrum for Minimum Rain Attenuations (Drizzle Conditions)

\section{CONCLUSIONS}

The performance of FSO system has been analyzed for two conditions (Heavy-Rain and Lighter Rain or Drizzle) at the heart of Kuala Lumpur in 2001. It concludes as follow:

1) Heavy rain with cloudburst had influenced the signal strength and produced higher rain attenuations.

2) Lighter rain is also influenced the signal strength but produced lower rain attenuations.

3) The attenuations values for heavy rain conditions at the heart of Kuala Lumpur city were -28.6dB.

4) The attenuations values for lighter rain conditions at the heart of Kuala Lumpur city were $-1.3 \mathrm{~dB}$.

5) FSO transmission at the heart of Kuala Lumpur city in heavy rain with cloudburst conditions was noticed to be BER $10^{-1}$.

6) FSO transmission at the heart of Kuala Lumpur city in lighter rain was noticed to be BER $10-^{13}$. 
7) FSO transmission at the heart of Kuala Lumpur city in heavy rain conditions was $-66.44 \mathrm{dBm}$.

8) FSO transmission at the heart of Kuala Lumpur city in lighter rain conditions was $-39.14 \mathrm{dBm}$.

\section{ACKNOWLEDGMENT}

I (Fatin Hamimi Mustafa) take this opportunity to thank my supervisor, Associate Prof Abu Sahmah Mohd Supa'at for his continuous support, patience, and also unlimited ideas during the experiments. A special thank is also delivered to Ministry of Science, Technology and Innovation (MOSTI) for financial support and also to the others who helped me directly or indirectly during the analysis and also compilation of this report.

\section{REFERENCES}

[1] Roberto Ramirez, Sevia M Idrus, Ziran Sun, Optical Wireless Communications: IR for Wireless Connectivity, $1^{\text {st }}$ edition, Broken Sound Parkway NW: CRC Press, 2008.

[2] Heinz Willebrand, Baksheesh S. Ghuman, Free Space Optics; Enabling Optical Connectivity in Today's Network, $1^{\text {st }}$ edition, Indiana, USA: Sams Publishing, 2002.

[3] Bouchet, H. Sizun, C. Boisrobert, F. Fornel, P. Favennec, Free Space Optics - Propagation and Communication, $1^{\text {st }}$ edition, Newport Beach, CA: ISTE, 2007.

[4] Propagation data required for the design of terrestrial free-space optical links, ITU-R Recommendation P.1817, 2007.

[5] Ahmed M. Mahdy and Jitender S. Deogun, "Optimizing Free Space Optics for City-Wide Wireless Networks," in Proceedings of the Sixth International Conference on Networking (ICN'07), 2007.

[6] Prediction methods required for the design of terrestrial free-space optical links, ITU-R Recommendation P.1814, 2007.

[7] A.K Rahman, M.S Anuar, S.A Aljunid and M.N Junita, "Study of rain attenuation consequence in free space optic transmission," in IEEE 6th National Conference on Telecommunication Technologies and Malaysia Conference on Photonics. 2008, pp. 26-29.

[8] (2010) Malaysian Meteorological Department Website. [Online]. Available: http://www.met.gov.my/

[9] Husagic Alma and Wajdi Al-Khateeb, "Effect of Weather Conditions on Quality of Free Space Optic Links (with focus on Malaysia)," in IEEE, International Conference on Computer and Communication Engineering, 2008, pp. 1206-1210.

[10] (2010) PWComms for FSO PAVLight product [Online]. Available: www.pavdata.com/en/products/

[11] G. Hansel, E. Kube, J.Becker, J. Haase and P. Schwarz, "Simulation in the Design Process of Free Space Optical Transmission System," in Proc. $6^{\text {th }}$ Workshop Optics in Computing Technology, Paderborn, Germany, 2003, pp. 45-53.

[12] Scott Bloom, Eric Korevaar, John Schuster and Heinz Willebrand, "Understanding the Performace of Free Space Optics," Jurnal of Optical Networking, Vol 2, No 6, June 2003.

[13] G. Shaulov, J. Patel, B. Whitlock, P. Mena and R. Scarmozino. "Simulated-Assisted Design of Free Space Optical Transmission Systems," RSoft Design Group, Ossining. NY, pp. 1-5.

[14] Bernard Sklar, Digital Communications: Fundamental and Applications, $2^{\text {nd }}$ Edition, Upper Saddle River, New Jersey: Prentice Hall, 2001.

[15] (2005) fSona Communication Corporation. Defining a Common Standard for Evaluating and Comparing Free-Space Optical Products. [Online]. Available: www.fsona.com/

[16] M. Al Nabousi, M. Sizun and de Fornell F, "Propagation of Optical and Infrared waves in the atmosphere," SPIE Journal, 2003, pp. 1-4. 